\title{
Bernard Conrad \\ Novel procedures for high-throughput analysis of a frequent insertion-deletion polymorphism in the human T-cell receptor beta locus
}

Received: 27 March 2004 / Revised: 26 April 2004 / Accepted: 26 April 2004 / Published online: 25 May 2004

(C) Springer-Verlag 2004

\begin{abstract}
The human T-cell receptor beta locus (TRB) contains two frequent insertion-deletion polymorphisms. In one, the insertion comprises two functional variable beta genes, TRBV6-2/TRBV6-3 and TRBV4-3, and the pseudogene TRBV3-2. Deletion of these TRBV genes may confer resistance and/or susceptibility to autoimmunity, analogously to findings in rodent models. Curiously, the TRBV domains in the insertion react with the HERV-K18 superantigen associated with type 1 diabetes. While this region has been extensively characterized before, typing methods compatible with high-throughput analysis are not yet available. Here, two novel procedures are reported that are suitable for large-scale association analysis of this polymorphism. One features a duplex TaqMan $5^{\prime}$-exonuclease assay that quantifies the gene dosage of TRBV3-2 present at 0,1 or 2 copies, with its closely related diploid relative TRBV3-1 as an internal reference, using the $2^{-\Delta \Delta C}$ T method. The other technique consists of two complementary long PCRs with primers specific for unique regions in the locus. The first discriminates individuals heterozygous or homozygous for the deletion, and the second, individuals heterozygous or homozygous for the insertion from other genotypes. These simple, solid, and cross-validated procedures can now be used in conjunction with flanking single-nucleotide polymorphisms for large-scale linkage studies.
\end{abstract}

B. Conrad

Department of Microbiology and Molecular Medicine, CMU,

University of Geneva,

1 rue Michel Servet,

1211 Geneva 4, Switzerland

B. Conrad $(\bowtie)$

Department of Genetic Medicine and Development, CMU,

University of Geneva,

1 rue Michel Servet,

1211 Geneva 4, Switzerland

e-mail: bernard.conrad@medecine.unige.ch

Tel.: +41-22-3795719

Fax: $+41-22-3795706$
Keywords T-cell receptor beta locus $\cdot$ Segmental duplication - Genome instability · Polymorphism • Autoimmunity

Human Chromosome 7 contains the largest number of intrachromosomal duplications (Scherer et al. 2003). In particular, the T-cell receptor beta (TRB) locus at 7q34 is composed of tandemly arranged gene segments, which have arisen by gene duplication events (Rowen et al. 1996), similar to other immunoglobulin (Lefranc and Lefranc 2001a), and T-cell receptor multigene loci (Lefranc and Lefranc 2001b). Segmental duplications are associated with genome instability and can cause aberrant recombination, which has emerged as an important factor in the causation of diseases associated with abnormal gene dosage (Emanuel and Shaikh 2001). In fact, one of those segmental duplications in the TRB locus subsequently generated a frequent insertion-deletion polymorphism (Fig. 1A) (Robinson et al. 1993; Seboun et al. 1989; Zhao et al. 1994). The inserted haplotype includes two functional TRBV genes, TRBV6-2/TRBV6-3, TRBV4-3, and one pseudogene, TRBV3-2 (Fig. 1A). While TRBV4-3 is unique, TRBV6-2 has another copy, TRBV6-3. The TRBV6-3 protein differs from TRBV6-2 by only one amino acid in the signal peptide, and therefore produces an identical surface protein (Arden et al. 1995). Because the breakpoint regions have not been unambiguously determined, this is consistent with two functionally equivalent deletion events, as indicated in Fig. 1A. The polymorphism quantitatively reflects on surface TRBV expression, in that individuals lacking the insert have no detectable TRBV4-3 expression, and consistently less cells express TRBV6-2/TRBV6-3 compared with inserted haplotypes (our unpublished data; Manavalan et al. 2004).

The TRBV insertion-deletion polymorphism is of interest for a number of reasons. Firstly, germline deletion of TRBV genes jointly with TRAV polymorphisms confers resistance to autoimmunity in certain rodent strains (Osman et al. 1999a, 1999b). Secondly, the two functional 
A 141.4MB

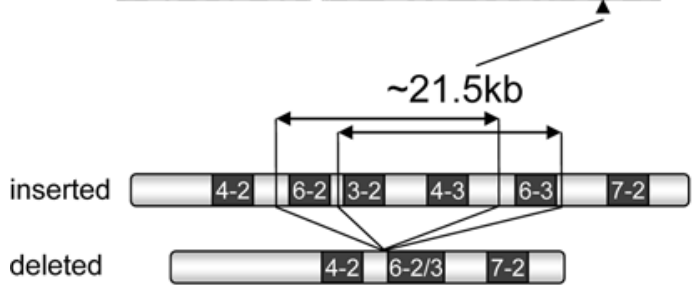

C

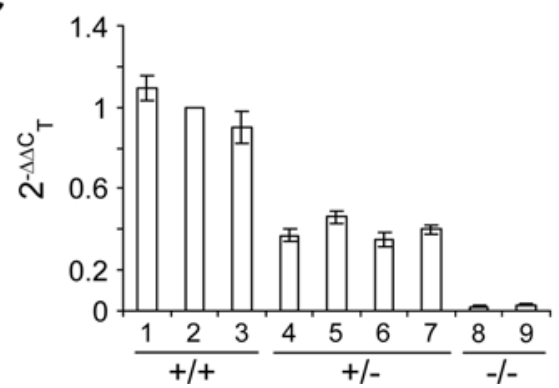

Fig. 1A-D Determining the zygosity of a frequent insertiondeletion polymorphism in the human TRB locus by quantitative duplex TaqMan analysis. A The human TRB locus at $7 \mathrm{q} 34$ $(141.4 \mathrm{Mb})$ contains a frequent insertion-deletion polymorphism. Inserted haplotypes have a unique TRBV4-3 copy, and two copies of TRBV6-2/6-3, while deleted haplotypes lack TRBV4-3, and have a single TRBV6-2/TRBV6-3 copy; TRBV3-2 is a pseudogene. The breakpoint regions have not been unambiguously mapped, which leaves two possible solutions open that are indicated separately. The TRBV6-3 protein differs from TRBV6-2 in only one amino acid in the signal peptide, producing an identical surface protein. B The summary of the results from all samples analyzed is shown. The $2^{-\Delta \Delta \mathrm{C}_{\mathrm{T}}}$ method yielded results consistent with the theoretical predictions for two, one and zero copies, namely $\sim 1, \sim 0.5$, and

$T R B V$ gene products derived from inserted haplotypes are reactive with the human endogenous retroviral (HERV)K18 superantigen (SAg) (Conrad et al. 1994, 1997; Stauffer et al. 2001; Sutkowski et al. 2001). Polymorphisms of the HERV-K18 SAg are associated with type 1 diabetes (T1D) (Marguerat et al. 2004). Therefore, deletion of the two TRBV genes could confer resistance, and/or susceptibility to T1D.

While considerable work has been carried out to characterize single-nucleotide polymorphisms (SNPs) and haplotype blocks in the TRBV locus (Mackelprang et al. 2002; Subrahmanyan et al. 2001), methods to determine all genotypes of the insertion-deletion polymorphism in a fashion compatible with high-throughput analysis are not yet available. Therefore, we developed two assays that could accomplish this and which crossvalidated each another. We quantified the insert gene dosage with TaqMan PCR, and specifically amplified the deleted and inserted regions by long PCR. Real-time quantitative PCR and the $2^{-\Delta \Delta C}$ method (Livak and Schmittgen 2001) have been exploited previously to determine the gene copy number associated with Chr 17 duplications and deletions (Thiel et al. 2003). We adopted a similar procedure for the TRBV insertion-deletion with some modifications. Initially, we aligned the three $T R B V$
B

\begin{tabular}{|cccc|}
\hline $\mathbf{N}$ & genotype & $\mathbf{2}^{-\Delta \Delta \mathrm{c}_{\mathrm{T}}}$ & {$[\%]$} \\
5 & $+/+$ & $\sim 1$ & 19.2 \\
16 & $+/-$ & $\sim 0.5$ & 61.5 \\
5 & $-/-$ & $<0.03$ & 19.2 \\
\hline
\end{tabular}

D

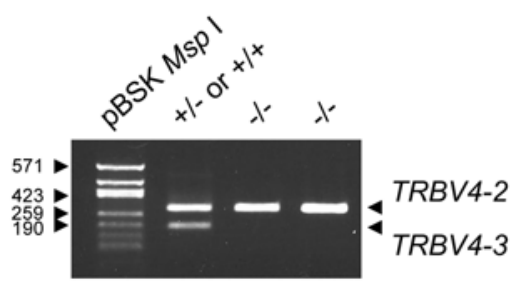

close to 0 for deletions, respectively. C Genomic DNA extracted from EBV-immortalized B cells was analyzed by duplex TaqMan analysis with primers and probes specific for TRBV3-2, and TRBV31 as diploid internal control. The mean $\pm 1 \mathrm{SD}$ of three independent experiments, each performed in quadruplicate for individual samples is shown. The value on the $y$-axis represents $2^{-\Delta \Delta C}$, a linear transformation of the differences in the threshold cycle between the diploid reference and samples. Sample 2 was set as the endogenous standard with reference value 1 . The genotypes are indicated below the sample numbers on the $x$-axis. D A previously reported conventional duplex PCR with primers specific for TRBV4-3/ TRBV4-2 (Charmley and Concannon 1995) was performed on samples that had been typed in $\mathbf{C}$

genes in the insertion with their closely related subgroup members. These alignments revealed that only TRBV3-2 diverged sufficiently from its diploid relative, TRBV3-1, to allow stringent discrimination of a small $\sim 150$-bp fragment. Accordingly, primers and probes within the most disparate sequences in this block were used in 5'exonuclease assays with the TaqMan chemistry. Two conditions had to be met for the $2^{-\Delta \Delta C}$ method to be valid (Livak and Schmittgen 2001). Firstly, we confirmed that the amplifications of TRBV3-1 and TRBV3-2 were equally efficient, by amplifying serially diluted templates and determining the respective slopes. Secondly, for the diploid internal control TRBV3-1 the limiting primer concentrations were determined, such that its amplification can be stopped before the reactants become limiting for the minority species TRBV3-2.

Thus, the $2^{-\Delta \Delta C}$ T procedure with primers specific for TRBV3-2/3-1 was as follows. Primers and probes were designed with the Primer Express software (Applied Biosystems). The primer sequences used were: TRBV3-1 forward, comprising codons 14-20 according to the IGMT unique numbering (Lefranc et al. 2003), 5'-AGATGGGAAACGACAAGTCCAT-3'; TRBV3-1 reverse, codons 71-58, 5'-GAACTGTTTCATTTATAATGAGCTCCTTAT-3'; TRBV3-2 forward, codons 13-20, 5'-ACA- 
GATGGGAAAAAAGGAGTCTCT-3'; TRBV3-2 reverse, codons 69-57, 5'-TTTCATTTAAAATTGGCTCCTTGTTA-3'. MGB probes were synthesized by Applied Biosystems UK. The TRBV3-1 probe spanning codons 27-31 (5'-CTGGGCCATGATACT-3') was VIC-labeled at the $5^{\prime}$ end; the TRBV3-2 probe comprising codons 27-37 (5'-TGGGCCATAATGCTA-3') was FAM-labeled at the 5' end. Real-time quantitative PCR was performed on an ABI PRISM 7700 Sequence Detection System (Applied Biosystems) using the reagents for quantitative PCR (qPCR Core kit, Eurogentec, Liege, Belgium) in 25- $\mu$ l reactions. Quadruplicates were run for each sample in 96well plates (Thermo-Fast 96, Abgene, Epsom, Surrey). A single Mastermix was prepared for individual plates, and comprised a $2 \times$ TaqMan Mastermix (Eurogentec, Belgium), and primer and probes in the concentrations given below. Limiting primer concentrations were determined for $T R B V 3-1$, and were $50 \mathrm{nM}$ for the forward primer, and $200 \mathrm{nM}$ for the reverse primer. The TRBV3-2 forward primer was used at $300 \mathrm{nM}$, the reverse primer at $200 \mathrm{nM}$; both probes were used at $200 \mathrm{nM}$. Genomic DNA in the range of 50-200 ng per well yielded robust results. After reagent distribution the plate was sealed with an adhesive cover, and was briefly centrifuged before analysis. The thermal cycling conditions were as follows, $95{ }^{\circ} \mathrm{C}$ for $10 \mathrm{~min}, 40$ cycles of $95{ }^{\circ} \mathrm{C}$ for $15 \mathrm{~s}$, and $60{ }^{\circ} \mathrm{C}$ for $1 \mathrm{~s}$. After the run, the data were analyzed by manually setting the $\mathrm{C}_{\mathrm{T}}$ threshold at different levels in the near linear range of the curve. The mean $\pm 1 \mathrm{SD}$ of the $\mathrm{C}_{\mathrm{T}}$ values was calculated for all quadruplicate wells, and a common $\mathrm{C}_{\mathrm{T}}$ threshold chosen for analysis based on the smallest SD. Subsequently the data were transformed into a linear form by the $2^{-\Delta \Delta C}$ equation, as described (Livak and Schmittgen 2001; Thiel et al. 2003). The final results are expressed as the mean $\pm 1 \mathrm{SD}$ of linear values obtained in multiple experiments.

With the $2^{-\Delta \Delta C}$ and other methods described, 26 genomic DNA samples were analyzed, and the results are summarized in Fig. 1B. In a first set of experiments the $2^{-\Delta \Delta \mathrm{C}} \mathrm{T}$ procedure was performed on all genomic DNA samples extracted from cord blood samples with a simple guanidine isothiocyanate-ethanol precipitation method (DNAzol, Invitrogen), according to the manufacturer's instructions. Concentrations were determined via $\mathrm{OD}_{260 / 280}$ on a spectrophotometer. Subsequently, these results were confirmed on genomic DNA extracted from EBV immortalized samples of the same individuals (Purgene, Gentra Systems, Minneapolis, according to the manufacturer's instructions) (Fig. 1C). This second method yielded DNA of higher purity and solubility compared with the first, since it stepwise involved cell lysis by sodium dodecyl sulfate-based detergents, RNAse A digestion and protein precipitation before DNA precipitation.

Five samples were homozygous for the insertion, 16 were heterozygous, and five homozygous for the deletion. The predictions of the $2^{-\Delta \Delta C}$ method were closely met, in that values of $\sim 1$ were obtained for samples with two copies of the insert, $\sim 0.5$ for one copy and values $<0.03$ for zero copies (Fig. 1C). The single most critical parameter was the quality of the genomic DNA, since discriminating two from one copy became difficult, although feasible with simple extraction procedures, such as those based on guanidine salts and ethanol precipitation only. As shown in Fig. 2D, a conventional duplex PCR for TRBV4-3 and TRBV4-2 previously described (Charmley and Concannon 1995) confirmed that samples that had been typed as having no copies of the insert with the $2^{-\Delta \Delta C}$ procedure and $T R B V 3-1 / T R B V 3-2$ primers were, as expected, also lacking TRBV4-3. While these results demonstrate that we could accurately discriminate samples homozygous for the deletion from other genotypes, additional methods had to be developed to corroborate the distinction of two from one insert copy.

The locus in and around the insertion-deletion polymorphism has undergone complex modifications that shaped its current structure (Fig. 2A). Most likely it evolved from one or more segmental duplications via unequal crossover, followed by deletions and gene conversion (Zhao et al. 1994). The almost perfectly conserved duplicated $\sim 6.6-\mathrm{kb}$ segments are shown with reference to the hypothetical breakpoint regions (L36092, IMGT/ LIGM-DB, http://imgt.cines.fr; Fig. 2A). The downstream duplicated sequence has undergone rearrangements compared with its upstream variant; namely, it acquired two small deletions and a novel EcoRI site. Discriminating the inserted from the deleted haplotype thus becomes feasible by long PCR using primers specific for unique regions outside the tandem repeats (Fig. 2B, C). The long PCR techniques were undertaken as follows. The positions of the hypothetical breakpoint regions were 139944-140442 for the upstream breakpoint, 161739-162237 for the downstream breakpoint (L36092, IMGT/LIGM-DB, http://imgt.cines.fr), and 3591-4088, respectively (L36190, IMGT/LIGM-DB). The region featuring the 114-nt deletion was located at 162287 in L36092, and at 4166 in L36190, compared with its expanded upstream homologue, 140504-140697 (L36092). The region containing the 23-nt deletion was located at position 166573 in L36092, at 8484 in L36190, respectively, and the expanded upstream region at position 144893-144915 (L36092). Primer sequences were as follows: for the 6.8$\mathrm{kb}$ PCR, the forward primer was 5'-GCTCATGTTTGTCTACAACTTTAAAGAACAGAC-3' (position 138376 138408 in L36092, and 2023-2055 in L36190, respectively), and the reverse primer 5'-AATATTTATAAGCTCCTTGTAAACAACGTTATTGCATGG-3'

(position 166994-167032 in L36092, and 8825-8862 in L36190, respectively); for the 9.3-kb PCR, the forward primer was 5'-TGACAGGAAATGTCACAGCCAATTGAATCTGC-3' (position 157594-157625 in L36092), and the reverse primer was identical to the one for the 6.8$\mathrm{kb}$ PCR. For the amplifications, 50-200 ng of genomic DNA was used with the Takara LA Taq, in 25- $\mu$ l reactions, according to the recommendations (http://www.takara-bio. co.jp/english/index.htm). The cycling conditions were as follows: $94{ }^{\circ} \mathrm{C}$ for $1 \mathrm{~min} ; 14$ cycles of $98{ }^{\circ} \mathrm{C}$ for $10 \mathrm{~s}$, $68{ }^{\circ} \mathrm{C}$ for $15 \mathrm{~min} ; 16$ cycles of $98^{\circ} \mathrm{C}$ for $10 \mathrm{~s}, 68^{\circ} \mathrm{C}$ for 
A

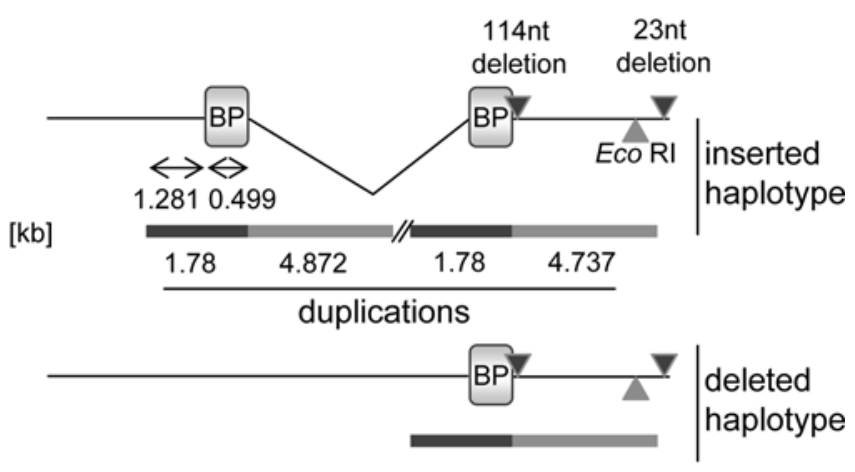

B
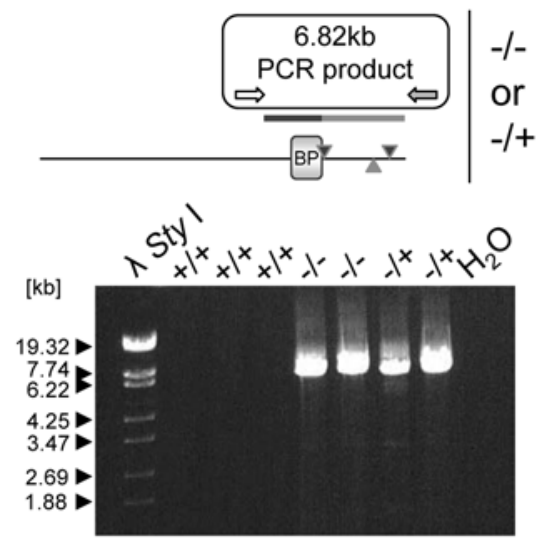

C
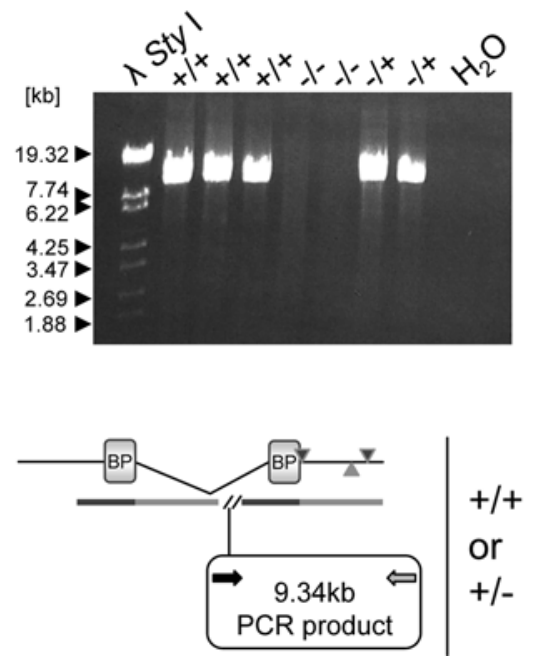

Fig. 2A-C Long PCR to determine the genotype of the insertiondeletion polymorphism. A The structure of the tandem repeats in the locus is shown with respect to the hypothetical breakpoints. The inserted and deleted haplotypes are designated in the assumption that the hypothetical breakpoints are used for deletion. The repeat sequence at and upstream of the hypothetical breakpoint are in blue, the repeat sequences downstream of the breakpoint are in red. The unique region between the repeats is denoted by two oblique lines. B Long PCR with primers annealing in the unique regions upstream and downstream of the repeats was performed on samples with the genotypes denoted on top of the gel. A $6.82-\mathrm{kb}$ product is expected from samples hetero- or homozygous for the deletion. C Long PCR with primers annealing in a unique insert region, and downstream of the repeats was performed on samples with the genotypes denoted on top of the gel. A 9.34-kb product is expected from samples hetero- or homozygous for the insertion
$15{ }^{\circ} \mathrm{C}$, plus a 15 -s autoextension/cycle; and $72{ }^{\circ} \mathrm{C}$ for $10 \mathrm{~min}$. The $6.8 \mathrm{-kb}$ product was verified by restriction analysis with EcoRI, PstI, and XbaI. The additional EcoRI site predicted in the downstream repeat was present as expected.

In this procedure, a common reverse primer was combined either with a primer annealing upstream of the first hypothetical breakpoint region (Fig. 2B), or with one annealing in the unique insert region between the repeats (Fig. 2C). A PCR product of $\sim 6.8 \mathrm{~kb}$ was exclusively generated from individuals with the deletion and was thus absent in individuals homozygous for the insertion (Fig. 2B). The $\sim 28$-kb PCR product expected for inserted haplotypes was not obtained, probably because of its size, and the complex template structure of this region. The second set of primers gave results complementary to the first (Fig. 2C). Samples with at least one copy of the insertion had $\sim 9.3-\mathrm{kb}$ products that were absent in individuals lacking the insert. The sequence identity of the PCR products was confirmed by restriction analysis. In sum, while this second set of techniques using long PCR cross-validated the results of the $2^{-\Delta \Delta \mathrm{C}}$ T procedure, they can also be used alone in assays requiring high-throughput analysis, because they are simple and robust.

Here the results of two novel procedures have been presented that allowed us to determine the copy number and the zygosity of a TRB insertion-deletion polymorphism, in a fashion compatible with the requirements for high-throughput analysis. As would be expected, the single most critical factor for consistency of both methods was a relatively pure genomic DNA sample. Subject to this condition, both methods were simple and robust. Poorly soluble genomic DNA negatively affected the accuracy of the duplex TaqMan assay, probably by generating inconsistencies in the DNA quantity between wells. As reported for a similar assay (Thiel et al. 2003), the test was robust for an input DNA range from $\sim 50$ to 200 ng. Combined with validated SNPs in this region (Mackelprang et al. 2002; Subrahmanyan et al. 2001), large-scale genotyping of the anticipated four haplotypes therefore becomes practicable. Such studies are motivated by both the predicted role of this region in conferring resistance or susceptibility to autoimmunity by analogy with animal studies (Osman et al. 1999a, 1999b), and based on the fact that the TRBV chains encoded by the insertion are reactive with the HERV-K18 SAg associated with T1D (Marguerat et al. 2004). While this manuscript was in preparation, a study reported that the homozygous deleted genotype of the polymorphism described here is associated with primary Sjögren's syndrome (Manavalan et al. 2004). This well illustrates the potential biological relevance of this polymorphism and its possible link with autoimmunity. Contrary to our approach, (1) this study did not directly genotype the inserted genes, but rather a polymorphic restriction site in a flanking gene, and qualitatively determined the presence or absence of the insert by simple PCR; (2) the genotype was deduced from the combination of these two variables, and not directly determined; (3) not all haplotypes were determined; (4) the 
procedure is not compatible with high throughput analysis. The assays presented here will therefore provide an invaluable asset that permits high throughput analysis of the biological role played by this interesting and complex region.

Acknowledgements This work was supported by the Swiss National Science Foundation, and the Helmut Horten Foundation. Cord blood cells were kindly supplied by Dr. J. Plum, Ghent University, and control DNA samples by Dr. D. Posnett, Cornell University Weill Medical College. We thank William Wang, Drs. J. Curran, Th. Köhler, and J. Todd for reading the manuscript and/or suggestions.

\section{References}

Arden B, Clark SP, Kabelitz D, Mak TW (1995) Human T-cell receptor variable gene segment families. Immunogenetics 42:455-500

Charmley P, Concannon P (1995) PCR-based genotyping and haplotype analysis of human TCRBV gene segment polymorphisms. Immunogenetics 42:254-261

Conrad B, Weidmann E, Trucco G, Rudert WA, Behboo R, Ricordi C, Rodriquez-Rilo H, Finegold D, Trucco M (1994) Evidence for superantigen involvement in insulin-dependent diabetes mellitus aetiology. Nature 371:351-355

Conrad B, Weissmahr RN, Boni J, Arcari R, Schupbach J, Mach B (1997) A human endogenous retroviral superantigen as candidate autoimmune gene in type I diabetes. Cell 90:303-313

Emanuel BS, Shaikh TH (2001) Segmental duplications: an 'expanding' role in genomic instability and disease. Nat Rev Genet 2:791-800

Lefranc MP, Lefranc G (2001a) The immunoglobulin factsbook. Academic Press, London

Lefranc MP, Lefranc G (2001b) The T cell receptor factsbook. Academic Press, London

Lefranc MP, Pommie C, Ruiz M, Giudicelli V, Foulquier E, Truong L, Thouvenin-Contet V, Lefranc G (2003) IMGT unique numbering for immunoglobulin and $\mathrm{T}$ cell receptor variable domains and Ig superfamily V-like domains. Dev Comp Immunol 27:55-77

Livak KJ, Schmittgen TD (2001) Analysis of relative gene expression data using real-time quantitative PCR and the 2(-Delta Delta C(T)) method. Methods 25:402-408

Mackelprang R, Carlson CS, Subrahmanyan L, Livingston RJ, Eberle MA, Nickerson DA (2002) Sequence variation in the human T-cell receptor loci. Immunol Rev 190:26-39

Manavalan SJ, Valiando JR, Reeves WH, Arnett FC, Necker A, Simantov R, Lyons R, Satoh M, Posnett DN (2004) Genomic absence of the gene encoding $\mathrm{T}$ cell receptor Vbeta7.2 is linked to the presence of autoantibodies in Sjogren's syndrome. Arthritis Rheum 50:187-198

Marguerat S, Wang WY, Todd JA, Conrad B (2004) Association of human endogenous retrovirus K-18 polymorphisms with type 1 diabetes. Diabetes 53:852-854
Osman GE, Hannibal MC, Anderson JP, Cheunsuk S, Lasky SR, Liggitt HD, Ladiges WC, Hood LE (1999a) T-cell receptor vbeta deletion and valpha polymorphism are responsible for the resistance of SWR mouse to arthritis induction. Immunogenetics 49:764-772

Osman GE, Hannibal MC, Anderson JP, Lasky SR, Ladiges WC, Hood L (1999b) FVB/N (H2(q)) mouse is resistant to arthritis induction and exhibits a genomic deletion of T-cell receptor $\mathrm{V}$ beta gene segments. Immunogenetics 49:851-859

Robinson MA, Mitchell MP, Wei S, Day CE, Zhao TM, Concannon $P$ (1993) Organization of human T-cell receptor beta-chain genes: clusters of $\mathrm{V}$ beta genes are present on chromosomes 7 and 9. Proc Natl Acad Sci USA 90:2433-2437

Rowen L, Koop BF, Hood L (1996) The complete 685-kilobase DNA sequence of the human beta $\mathrm{T}$ cell receptor locus. Science 272:1755-1762

Scherer SW, Cheung J, MacDonald JR, Osborne LR, Nakabayashi K, Herbrick JA, Carson AR, Parker-Katiraee L, Skaug J, Khaja R, Zhang J, Hudek AK, Li M, Haddad M, Duggan GE, Fernandez BA, Kanematsu E, Gentles S, Christopoulos CC, Choufani S, Kwasnicka D, Zheng XH, Lai Z, Nusskern D, Zhang Q, Gu Z, Lu F, Zeesman S, Nowaczyk MJ, Teshima I, Chitayat D, Shuman C, Weksberg R, Zackai EH, Grebe TA, Cox SR, Kirkpatrick SJ, Rahman N, Friedman JM, Heng HH, Pelicci PG, Lo-Coco F, Belloni E, Shaffer LG, Pober B, Morton CC, Gusella JF, Bruns GA, Korf BR, Quade BJ, Ligon AH, Ferguson H, Higgins AW, Leach NT, Herrick SR, Lemyre E, Farra CG, Kim HG, Summers AM, Gripp KW, Roberts W, Szatmari P, Winsor EJ, Grzeschik KH, Teebi A, Minassian BA, Kere J, Armengol L, Pujana MA, Estivill X, Wilson MD, Koop BF, Tosi S, Moore GE, Boright AP, Zlotorynski E, Kerem B, Kroisel PM, Petek E, Oscier DG, Mould SJ, Dohner H, Dohner K, Rommens JM, Vincent JB, Venter JC, Li PW, Mural RJ, Adams MD, Tsui LC (2003) Human chromosome 7: DNA sequence and biology. Science 300:767-772

Seboun E, Robinson MA, Kindt TJ, Hauser SL (1989) Insertion/ deletion-related polymorphisms in the human $\mathrm{T}$ cell receptor beta gene complex. J Exp Med 170:1263-1270

Stauffer Y, Marguerat S, Meylan F, Ucla C, Sutkowski N, Huber B, Pelet T, Conrad B (2001) Interferon-alpha-induced endogenous superantigen. a model linking environment and autoimmunity. Immunity 15:591-601

Subrahmanyan L, Eberle MA, Clark AG, Kruglyak L, Nickerson DA (2001) Sequence variation and linkage disequilibrium in the human T-cell receptor beta (TCRB) locus. Am J Hum Genet 69:381-395

Sutkowski N, Conrad B, Thorley-Lawson DA, Huber BT (2001) Epstein-Barr virus transactivates the human endogenous retrovirus HERV-K18 that encodes a superantigen. Immunity 15:579-589

Thiel CT, Kraus C, Rauch A, Ekici AB, Rautenstrauss B, Reis A (2003) A new quantitative PCR multiplex assay for rapid analysis of chromosome 17p11.2-12 duplications and deletions leading to HMSN/HNPP. Eur J Hum Genet 11:170-178

Zhao TM, Whitaker SE, Robinson MA (1994) A genetically determined insertion/deletion related polymorphism in human $\mathrm{T}$ cell receptor beta chain (TCRB) includes functional variable gene segments. J Exp Med 180:1405-1414 